\title{
Kinetic and Process Modeling of Thermal and Mechanical Degradation in Ultrahigh Speed Twin Screw Extrusion
}

\author{
Azadeh Farahanchi ${ }^{1}$, Margaret J. Sobkowicz ${ }^{1}$ \\ ${ }^{1}$ Department of Plastics Engineering, University of Massachusetts \\ Lowell, One University Avenue, Lowell, Massachusetts 01854
}

Correspondence to:

Name: Margaret J. Sobkowicz

Telephone: 978-934-3433

E-mail Address: margaret_sobkowiczKline@uml.edu

Postal Address: 1 University Avenue, Lowell, Massachusetts

01854, Plastics Engineering Department, Ball 121, University of

Massachusetts Lowell

(C) 2017. This manuscript version is made available under the Elsevier user license http://www.elsevier.com/open-access/userlicense/1.0/ 


\section{ABSTRACT}

In this article, a kinetic model is introduced in conjunction with experimental extrusion results to predict the evolution of depolymerization reaction at various operating conditions during ultra-high speed twin screw extrusion (TSE). A commercially available flow simulation software $\left(\right.$ LUDOVIC $\left.{ }^{\circledR}\right)$ was used to calculate thermomechanical flow parameters for various screw profiles. The simulated local material temperature and mechanical shear rate were used as inputs to develop a kinetic model that separately describes the effects of temperature and shear on the extent of degradation reaction due to extruding at very high speeds. The model is validated through comparison with experimental gel permeation chromatography (GPC) results on extruded samples. A good agreement was found between theoretical and experimental results. This validation makes the model practically useful for processing optimization, which is a considerable challenge for realizing compounding benefits in ultra-high speed TSE.

KEY WORDS: reactive extrusion, high speed twin screw extrusion, degradation, mechanical shear, modeling. 


\section{INTRODUCTION}

Extrusion systems are increasingly being used as continuous chemical reactors to perform modification on polymers and develop new materials. Reactive extrusion has found applications in polymerization, dynamic vulcanization, rheological and chemical modification of plastics such as adjusting the viscosity of the resins to the desired level, functionalization, cross linking and grafting [1-3]. Reactive extrusion is of industrial importance because it is solvent-free, continuous, scalable and highly flexible for a range of materials and operating conditions. Among all the extrusion techniques, twin screw extrusion (TSE) is the most attractive method for reactive processes due to the modular geometry of screw-barrel blocks, great flexibility in the design of screw profiles, exceptional mixing capabilities in viscous media and control over the mixing conditions according to the geometry of the kneading block sections [4-6].

The co-rotating, fully intermeshing, self-wiping, ultra-high speed TSE used in this study has the capability of rotating much faster than typical equipment, as high as 4500 RPM. This results in remarkably high shear rate and mechanical energy imparted to the extruded materials, which is expected to increase the mixing efficiency. On the other hand, this specific condition makes the control and monitoring of chemical reactions inside the extruder 
even more challenging. Depolymerization and other unintentional conversions along the extruder while running at very high speeds may adversely affect material properties and final product performance.[7] Previous works have studied the thermomechanical degradation of thermoplastics in conventional extrusion processes; however, almost none of these works concern the very high shear stress encountered in modern polymer processes, and most do not attempt to separate thermal and mechanical factors causing degradation. Silvano et al. observed decreased viscosity in neat polypropylene as well as $\mathrm{PP} /$ montmorillonite nanocomposites when subjected to multiple reprocessing cycles in a TSE. They related it to the thermal chain scission and molecular weight reduction [8]. Likewise, Remili et al. observed a large reduction of molecular weight of neat polystyrene with increasing the number of reprocessing cycles due to chain scission through a series of eight extrusion cycles [9]. Saw et al. studied processing-induced degradation of polypropyleneethylene copolymer-kaolin composite in TSE using FTIR measurements. They recognized that the filler loading accelerated the degradation process due to scission of high molecular weight chains. However, they did not observe noticeable rheological behavior changes [10]. Yousfi et al. observed brittle behavior of the nanofilled polyethylene terephthalate/polyethylene (PET/PE) 
blends processed in TSE compared to the neat blend as a result of thermal degradation of the clay organomodifier during compounding [11]. Mohamed et al.[12], Eise et al.[13], and Altomare et al.[14] all predicted that screw speed and throughput are the main variables affecting degradation.

The ultra-high speed capability of the TSE examined in this work gives rise to questions regarding negative effects of such extreme mechanical forces on polymers. In order to optimize compounding operations, an accurate prediction of depolymerization kinetics is necessary. Further, accurate prediction of any desired reaction depending on process variables such as screw speed, screw profile and temperature will allow better control over the wide variety of extrusion operations that may benefit from high shear. Fluid dynamics simulation software is widely available that can predict the main flow parameters (such as average temperature, pressure, residence time, and shear rate) down the length of the screw, and the interactions between these parameters and the numerous operating conditions. Previous works have performed theoretical modeling/thermomechanical calculations of reactive systems in TSE [15-17]. Their models have been used by other researchers for many extrusion reaction applications including esterification of ethylene vinyl acetate (EVA) copolymer [18], polymerization of $\varepsilon$ caprolactone [19], starch cationization reaction [20], and the 
peroxide-induced degradation of polypropylene along a TSE [2123]. However, in all these works the extrusion screw speed was not beyond 600 RPM. As a result, they did not consider the effect of mechanical shear rate as a separate important factor on the evolution of the reaction. Most of the previous models reported that the extent of the reaction follows the evolution of only temperature and residence time.

In order to theoretically predict the progress of the reaction inside the ultra-high speed TSE, first we need thermomechanical representation of the main flow parameters along the barrel using simulation software. Then we need to define kinetic equations controlled by kinetic constants that take into account the expected chemical reactions (in our case, chain scission degradation) $[24,25]$. Our previous work on the ultra-high speed TSE differentiated between the thermal and mechanical stress contributions to degradation of polymers running at high speeds with one screw configuration [26]. We found that a large effect not accounted for by temperature alone is at play which is not particularly relevant at conventional equipment with normal operating screw speeds. The purpose of this work is to derive and test a predictive kinetic model of the evolution of conversion along various screw profiles that takes into account the thermal and mechanical shear stress factors separately. Specifically, we 
compare the molecular weight ratio of extruded polystyrene at different locations along the axis of the ultra-high speed TSE with model predictions. Thereby, for any specific industrial application, one is able to predict the optimal operating conditions to best mitigate degradation, and develop or modify new polymeric materials with desired final qualities.

\section{EXPERIMENTAL}

\subsection{Material}

The base polymer evaluated in this study was an injection molding grade polystyrene (PS) MC3650 with a melt flow rate (MFR) of 13 $\mathrm{g} / 10 \mathrm{~min}$ at $200{ }^{\circ} \mathrm{C} / 5 \mathrm{~kg}$. The material was supplied by Amsty Styrenics Corporation.

\subsection{TSE Experiments}

This study employed a co-rotating, fully intermeshing, self-wiping, ultra-high speed twin screw extruder manufactured by the TECHNOVEL Corporation of Osaka Japan, KZW 15TW, with the following characteristics: top screw speed of 4500 RPM, screw diameter of $15 \mathrm{~mm}$ and length to diameter ratio of 60:1. The TSE consisted of 8 barrel zones along with a single head die, strand die and a water cooling trough. Reactive experiments were conducted by varying screw speed at 1000 RPM, 2000 RPM, 3000 RPM, and 4000 RPM with a uniform flat profile barrel/die set temperature of 
$210{ }^{\circ} \mathrm{C}$. PS was fed into the main hopper located at the first barrel zone using a volumetric feeder (5IK90GU-SWT, ORIENTAL MOTOR CO.,LTD.) at a constant rate of $27 \mathrm{~g} / \mathrm{min}$. The experiments were repeated for three different screw profiles. After reaching steady state conditions for each run, samples were collected at four locations along the screw axis: $320 \mathrm{~mm}, 520 \mathrm{~mm}$, and $720 \mathrm{~mm}$ and at the die exit. The actual melt temperature was measured using a sheathed type $\mathrm{k}$ immersion thermocouple extending $6 \mathrm{~mm}$ into the melt stream in the die adaptor located just before the strand die. The minimum residence time was measured using colored pellets dropped directly into the main hopper at the feed zone for each processing condition. The time between delivery of the colored pellet to the hopper and the first trace of color change in the extruded samples coming out from the die provided the minimum residence time. The TSE was programmed with two-flighted screw elements at various pitches and lengths as well as different kneading blocks in the mixing zones (see supporting information for detailed descriptions of the screw programs). The first screw profile provided the lowest shear. It included of simple forward flight conveying elements along with only two backward flight reverse elements inserted right before the vent ports. Figure 1 shows the schematics representing the extruder at each screw profile in this study as well as the locations of 
sample collections. The downward arrows represent the feeder location at the beginning of the extruder and the upward arrows represent the venting ports located right after the reverse screw elements in barrel zone 1 and barrel zone 7 . The second screw profile had an intermediate level of shear. In this profile six kneading blocks (with 5 kneading discs, staggering angle of $45^{\circ}$ for right hand side and left hand side KBs and staggering angle of $90^{\circ}$ for neutral $\mathrm{KBs}$ ) were added. The third screw profile imparted the most aggressive shear on the melt. In this profile in addition to the mixing zones described for profile 2, six more KBs were added in the plastication section of the extruder.
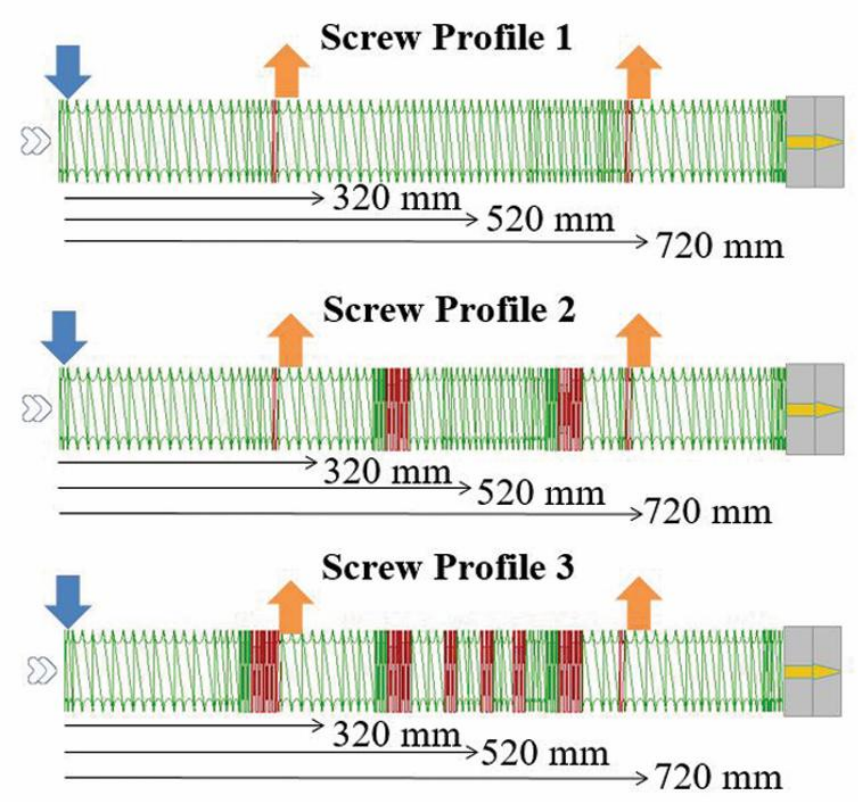

FIGURE 1. Extrusion set-ups and sampling locations for various screw profiles. 


\subsection{Molecular Weight Measurements}

Molecular weight measurements were made using a Waters Alliance 2695 Gel Permeation Chromatograph (GPC) and a 2410 differential refractometer. Three Waters Styragel HR GPC columns (gel size: $5 \mu \mathrm{m}$, column diameter: $7.8 \mathrm{~mm}$, and column length: $300 \mathrm{~mm})$ were connected in the order of: HR $4\left(M_{w}\right.$ range of $5000-600000 \mathrm{~g} / \mathrm{mol})$, HR $3\left(M_{w}\right.$ range of $\left.500-20000 \mathrm{~g} / \mathrm{mol}\right)$, and HR $1\left(M_{w}\right.$ range of $\left.100-5000 \mathrm{~g} / \mathrm{mol}\right)$. The eluent was roomtemperature tetrahydrofuran iso-cratic at a flow rate of 0.9 $\mathrm{mL} / \mathrm{min}$. To prepare the samples, 2 milligrams of each sample was dissolved in $2 \mathrm{~mL}$ tetrahydrofuran.

\subsection{TSE Simulation Methods}

As mentioned previously, one of the necessary elements for modeling a reactive extrusion is a tool for description and computation of the thermomechanical flow conditions along the complex geometry of TSE [27]. For this purpose, we used commercial LUDOVIC ${ }^{\circledR}$ software developed by "Sciences Computers Consultants Company" (Saint-Etienne France). This software uses a global model based on local one-dimensional approximations, which computes the main thermomechanical flow parameters including average melt temperature, residence time, local shear rate, and filling ratio along the screw profile from the hopper to the die exit [28]. The simulation assumes non-isothermal 
flow conditions to determine the temperature profile. It also calculates the local shear rate from geometry of extruder and screw rotational speed [24]. The calculation of residence time for restrictive elements (where the polymer melt resides longer) is based on the ratio of the volume occupied by the molten polymer over the total flow rate [29]. The material parameters in the simulation were for a predefined PS with the same viscosity-shear relationship as our grade, with melting temperature of $130{ }^{\circ} \mathrm{C}$. The product was defined by its thermal characteristics as follows: specific heat capacity at $1838 \mathrm{~J} / \mathrm{kg}{ }^{\circ} \mathrm{C}$, melt density at $950 \mathrm{Kg} / \mathrm{m}^{3}$, and melt thermal conductivity at $0.128 \mathrm{~W} / \mathrm{m} . \mathrm{K}$. Non-isothermal flow conditions including dissipated power and heat transfer (conduction exchange toward the barrel and screws via Fourier model) have been assumed to calculate the local thermal energy balance. Heat transfer coefficient for the die and barrel was constant at $500 \mathrm{~W} / \mathrm{m}^{2} . \mathrm{K}$ for all the processing conditions. The rheological behavior of the product was pre-defined by a power law model with Arrhenius temperature dependence as follows:

$\eta=K_{0} \exp \left[E_{a} / R\left(1 / T-1 / T_{0}\right)\right] \dot{\gamma}^{m-1}$

where power law index $(m)$ was defined at 0.374 , consistency $\left(K_{0}\right)$ was defined at $6876 \mathrm{~Pa} . \mathrm{s}$, Reference temperature $\left(T_{0}\right)$ was defined at $180{ }^{\circ} \mathrm{C}$, and activation energy $\left(E_{a} / R\right)$ was defined at $3410 \mathrm{~K}$ specifically for the material. The extruder characteristics were 
defined as follows: diameter of $15.5 \mathrm{~mm}$, screw/barrel leakage of $0.1 \mathrm{~mm}$, centerline distance of $12.3 \mathrm{~mm}, 8$ barrel zones with the length of $110.6 \mathrm{~mm}$ of each and total length of $885 \mathrm{~mm}$. The head die was assumed as an annular convergent with length $35 \mathrm{~mm}$. The die was modeled as a converging pipe with length of $35 \mathrm{~mm}$, entrance diameter of $20 \mathrm{~mm}$ and exit diameter of $2.6 \mathrm{~mm}$.

\section{RESULTS AND DISCUSSION}

The first task was to confirm that the CFD simulation is able to accurately estimate the main thermomechanical process parameters without considering reaction in the ultra-high speed conditions. Computed and measured values of melt temperature and residence time were compared. These quantities are the independent variables in the reaction kinetic model. Figure 2 provides a direct comparison between computed and measured exit melt temperature and minimum residence time at different screw speeds and different screw profiles. All the experimental measurements have been done at a constant feeder rate and a constant barrel set temperature as described in the experimental section. 
a)

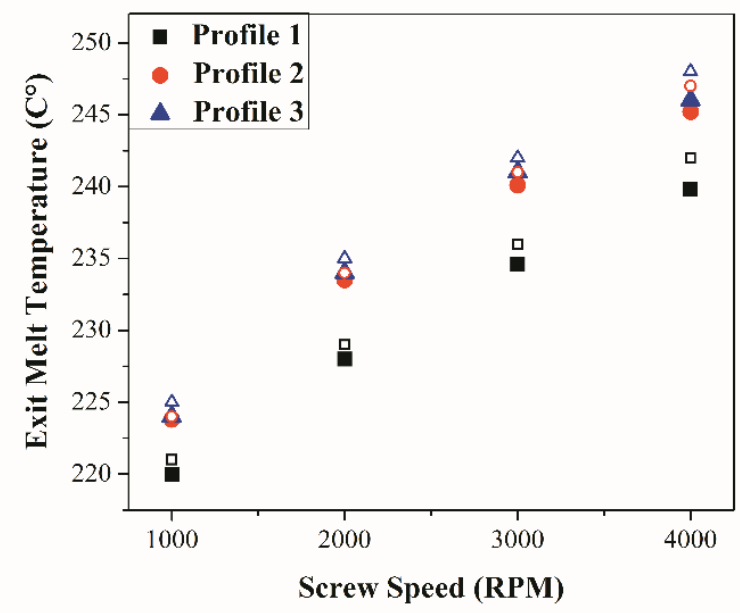

b)

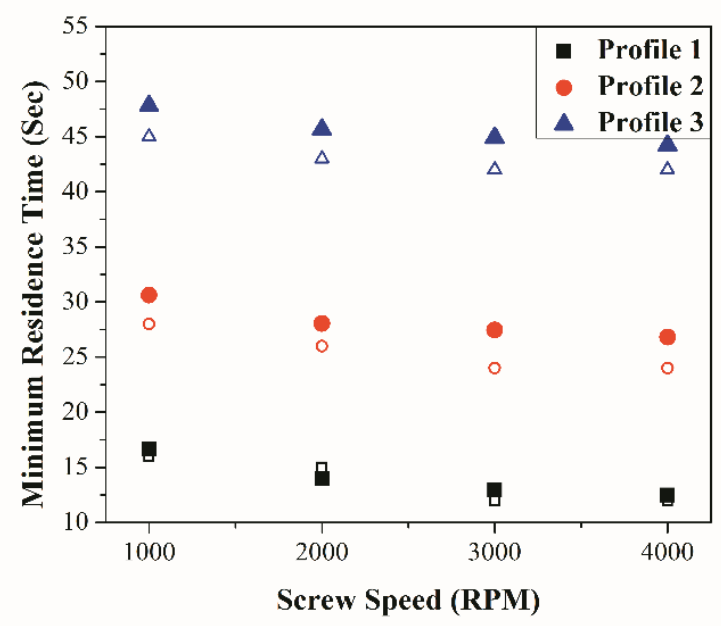

FIGURE 2. Comparison between computed (solid symbols) and measured (open symbols) of a) exit melt temperature and b) minimum residence time at various screw profiles.

As shown in Figure 2, all the computed results are within the $\pm 0.5 \%$ range of measured values for melt temperature and within 
the $\pm 6 \%$ range of measured values for residence time. The graphs clearly show the increasing melt temperature with increasing screw speed due to mechanical shear and viscous dissipation. The minor disparity between simulated and measured melt temperature can be attributed to the simplified melting mechanism assumption in the software. It is prescribed that melting is instantaneous and that it takes place before the first restrictive element of the screw profile. Another source of uncertainty could be related to the variance in heat transfer coefficient. LUDOVIC ${ }^{\circledR}$ calculations consider heat transfer coefficient to be a fixed value; however, it actually varies with the screw speed and the position along the screws [24]. Despite the slight differences, in all cases the software was able to closely estimate temperature and residence time at high speeds and provided good agreement with the measured values. Disparity in minimum residence time could be due to leakage from minor screw wear or imperfections. Moreover, as observed in Figure 2b the residence time has a minor dependence on the screw speed. This is because residence time is chiefly dictated by feeder rate in starved operation. Poulesquen et al. showed that the effect of screw speed on the residence time is minimized as the screw speed increases [29]. The computed results at the ultra-high screw speeds qualitatively showed the same trend. 
Figure 3 illustrates the temperature profile along the three different screw programs at a constant feeder rate of $27 \mathrm{~g} / \mathrm{min}$ and screw speed of 4000 RPM. As observed, the incorporation of kneading blocks in the profile 2 and profile 3 provides higher mechanical energy and viscous heating which leads to higher resin melt temperature through the length of the barrel.

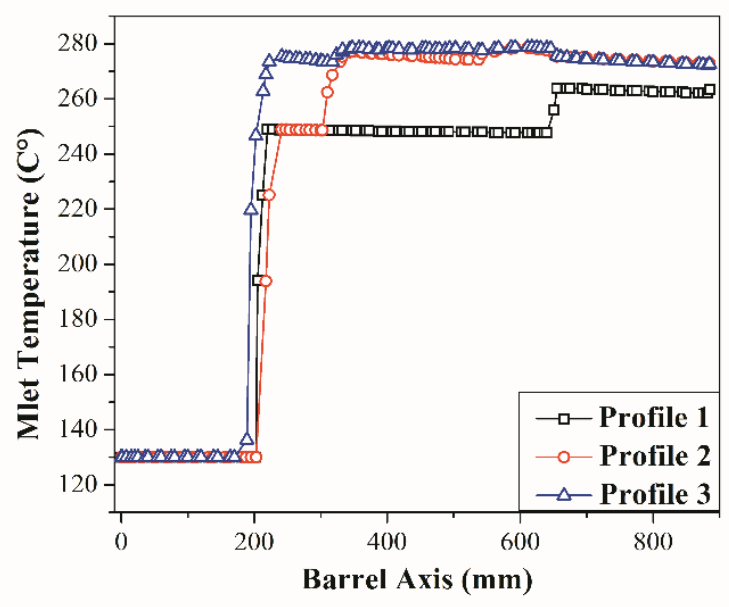

FIGURE 3. Temperature profile along different screw profiles at a constant feeder rate and screw speed.

Figure 4 shows the residence time distribution (RTD) for the three screw profiles at a constant feeder rate of $27 \mathrm{~g} / \mathrm{min}$ and screw speed of 4000 RPM. The breadth of the residence time distribution depends on the amount of flow restriction in the form of kneading elements. These restricted filled sections are characterized by a local increase of average residence time, which affects the extruder energy and reaction development in these sections. The residence 
time distribution is shifted towards higher times with replacement of conveying elements with kneading blocks in profile 2 and profile 3 as well as an important increase of broadness of distribution indicating improved axial mixing.

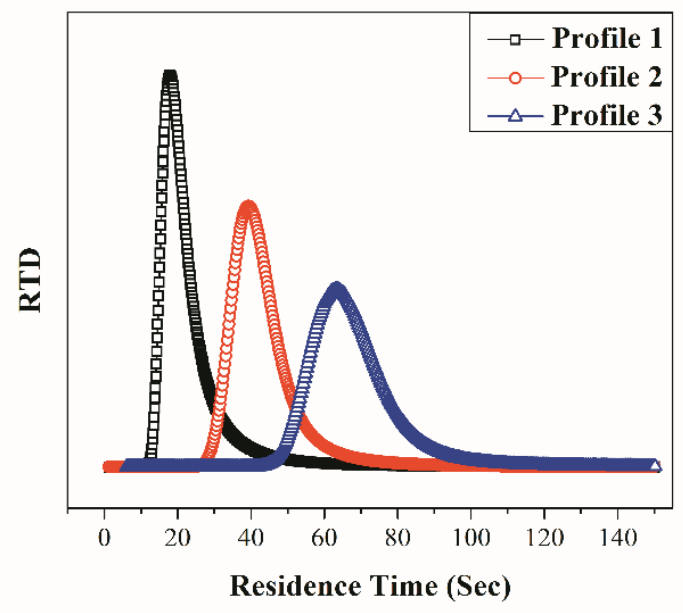

FIGURE 4. RTD for different screw profiles at a constant feeder rate and constant screw speed.

These simulations clearly indicate that the screw profile is an important factor influencing the thermomechanical parameters and consequently reaction development in TSE. The kneading block sections, which are characterized by a local increase in average residence time, increase the extruder power consumption and increase both the intensity and duration of shear experienced by the polymer melt. As a result, it is apparent that the screw profile plays a significant role on the reaction development in TSE. 
Previous studies predicted that the average shear rate in TSE can be defined as a function of screw speed and parameters related to the extruder program [30,31]. In this study, we have used this definition in order to quantitatively characterize various types of the screw profile inside the ultra-high speed extrusion as follows:

$\dot{\gamma}=C .2 \pi . N / 60$

Where $N$ is screw speed in RPM, $C$ is a dimensionless parameter, defined as the screw constant. It is fixed for a given configuration and is related to the extent of a given reaction inside the extrusion. $\dot{\gamma}$ is the position average shear rate in $\mathrm{s}^{-1}$, defined as:

$\dot{\gamma}=\left(\sum \dot{\gamma}_{i} \times x_{i}\right) /\left(\sum x_{i}\right)$

where $\dot{\gamma}_{i}$ is the local shear rate exerted on the polymer for a given element of length $x_{i}$. The values for the local shear rates have been provided by the simulation based on the type of the element and predefined screw element/kneading block geometries. In order to extract the characteristic constant $\mathrm{C}$ for each screw profile, we accumulated the given values of local shear rates and set $\sum_{i} x_{i}$ equal to $885 \mathrm{~mm}$ (total length of the barrel). We plotted this model for the three screw profiles in this work and used a linear curve fitting routine of shear rate versus screw speed to calculate the number of the extruder constant or $C$ parameter for each screw profile. 


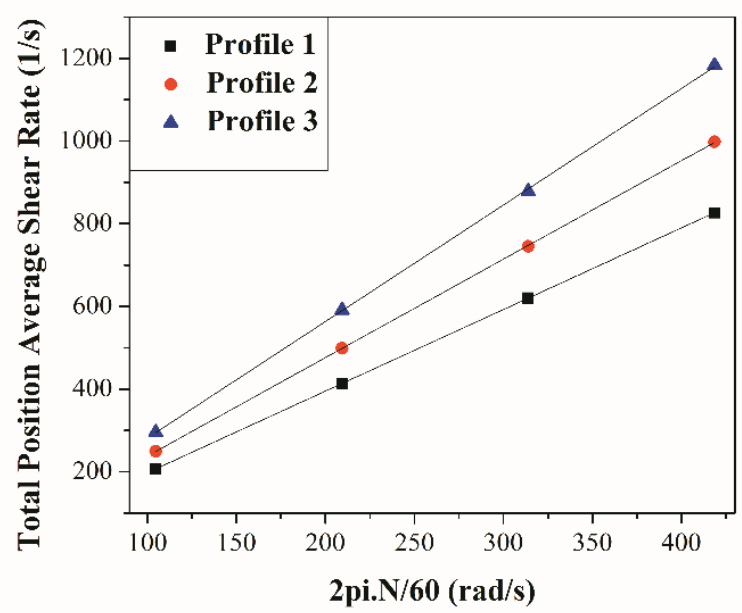

FIGURE 5. Total position average shear rate changes as a function of screw speed at various profiles.

Table 1 provides the dimensionless extruder constant values for each screw profile.

TABLE 1. Extruder constant values for various screw profiles.

\begin{tabular}{|c|c|c|}
\hline Screw Profile & $\begin{array}{c}\text { Extruder } \\
\text { Constant }\end{array}$ & $\begin{array}{c}\text { Coefficient of } \\
\text { correlation (R) }\end{array}$ \\
\hline Profile 1 & 1.94 & 1 \\
\hline Profile 2 & 2.38 & 0.99 \\
\hline Profile 3 & 2.81 & 0.99 \\
\hline
\end{tabular}

As shown in Table 4, the screw profile not only affects the thermomechanical flow parameters (local melt temperature rise, residence time, and shear rate at restrictive elements) but also the maximum shear rate and its dependence on screw speed (the slope value that has been considered as extruder constant). This 
parameter is unique for a given screw configuration and increases with the number of the restrictive elements in the profile. All this information will lead to a more accurate estimation of reaction in ultra-high speed TSE.

\subsection{Depolymerization Reaction Kinetic Model}

In order to describe the extent of reaction, a simple kinetic model was employed that predicts the decrease in molecular weight ratio along the axis of ultra-high speed TSE. The dominant mechanism of depolymerization is formation of free radicals and chain scission for polystyrene. The initiation step of this reaction can be defined by a simplified kinetic scheme as follows:

$P_{n} \rightarrow R_{r}+R_{n-r}$

where $P_{n}$ represents the polymer with $\mathrm{n}$ monomer units and $R_{r}$ is a radical containing $\mathrm{r}$ monomer units. The rate of this reaction can be represented by:

$d P / d t=-k_{d}[P]$

where $k_{d}$ is the kinetic constants for depropagation reaction.

An exponential equation is obtained through integration (6).

$[P]=[P]_{0} \exp \left(-k_{d} t\right)$

In the previous study on the ultra-high speed TSE, we concluded that high mechanical shear rate at high rotational screw speeds 
contributes significantly to the molecular weight reduction of PS resins and it dominates over thermal effects [26]. Our previously derived kinetic model represented the thermal effects and mechanical shear effects with two separate depropagation constants $\left(k_{d 1}\right.$ and $\left.k_{d 2}\right)$ for the thermal and mechanical contributions, respectively. The thermal rate constant, $k_{d 1}$ can be classically defined using the Arrhenius equation, Equation 7. The mechanical shear rate constant, $k_{d 2}$, is represented as an exponential at shown in Equation 8.

$k_{d 1}=A \exp \left(-E_{a} / R T\right)$

$k_{d 2}=\dot{\gamma} \exp (-1 / C)$

In equation (7), $\mathrm{T}$ is the average local melt temperature along the screw axis, $E_{a}$ is the activation energy (the minimum energy required for the reaction to occur), $R$ is the universal gas constant $\left(8.314 \times 10^{-3} \mathrm{~kJ} / \mathrm{mol} . \mathrm{K}\right)$, and $\mathrm{A}$ is the pre-exponential factor (prefactor). In equation (8), $\dot{\gamma}$ is the position average shear rate along the screw axis (calculated using Equation (3) at each position along the barrel), and $C$ is the extruder constant which can be defined according to the given screw profile

Equation (8) represents a constitutive relation for the effect of mechanical shear rate and screw profile on the kinetic constant for the depolymerization reaction. We selected an equation form that 
mimics the temperature dependence commonly used in kinetic rate constant expressions. In this equation, $\dot{\gamma}$ is pre-exponential factor and shows the average amount of the shear rate at each screw position, also depending on screw speed. Increasing the shear rate or screw speed speeds up the reaction rate. (Same as $\mathrm{A}$ in the Arrhenius equation, which is the pre-exponential factor for each chemical reaction and defines the rate of the reaction). Also included in the constitutive relation is the effect of the screw profile on the reaction. More aggressive screw profiles favor larger rate constants and as a result speed up the reaction. This dependency can be defined as an exponential relationship $\exp (-1 / C)$, as for very high number of restrictive elements $(C$ goes to infinity) the degradation tends to level off and approaches a limit. This can be equivalent to the temperature dependency of the reaction in Arrhenius equation. According to the Arrhenius equation, the kinetic constant increases as the temperature increases. Initially the kinetic constant increases exponentially however at very high temperatures its value approaches a limit.

The total depropagation kinetic constant for the reaction is defined by multiplying these two separate parameters:

$$
k_{d}=k_{d 1} \times k_{d 2}
$$

Or in a $\log$ form, 
$\operatorname{Ln}\left(k_{d}\right)=\operatorname{Ln}\left(k_{d 1}\right)+\operatorname{Ln}\left(k_{d 2}\right)$

The complete expression for depropagation reaction kinetic constant is then:

$\operatorname{Ln}\left(k_{d}\right)=\left(-E_{a} / R T\right)+\operatorname{Ln}(A)+(-1 / C)+\operatorname{Ln}(\dot{\gamma})$

We validated the accuracy of the model by systematic comparison between the numerically assessed depropagation reaction ratio and the experimentally measured molecular weight ratio. Figure 4 illustrates the comparison for the three screws studied here at different screw speeds.

a)

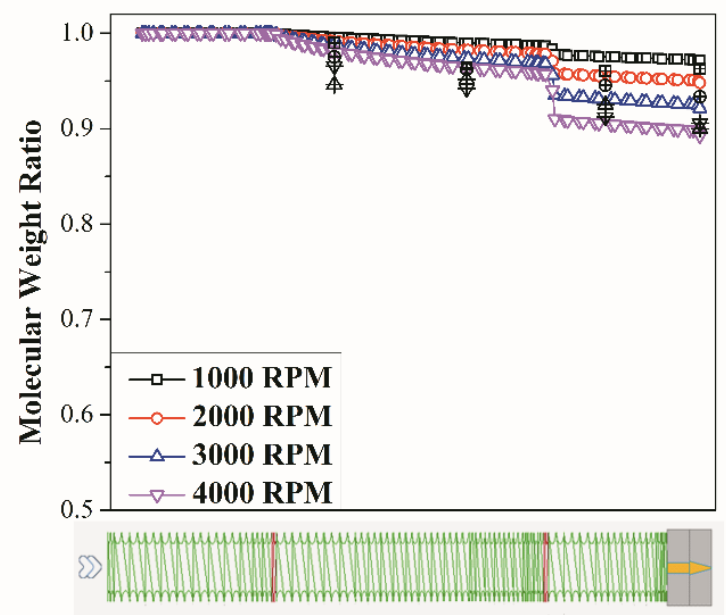


b)

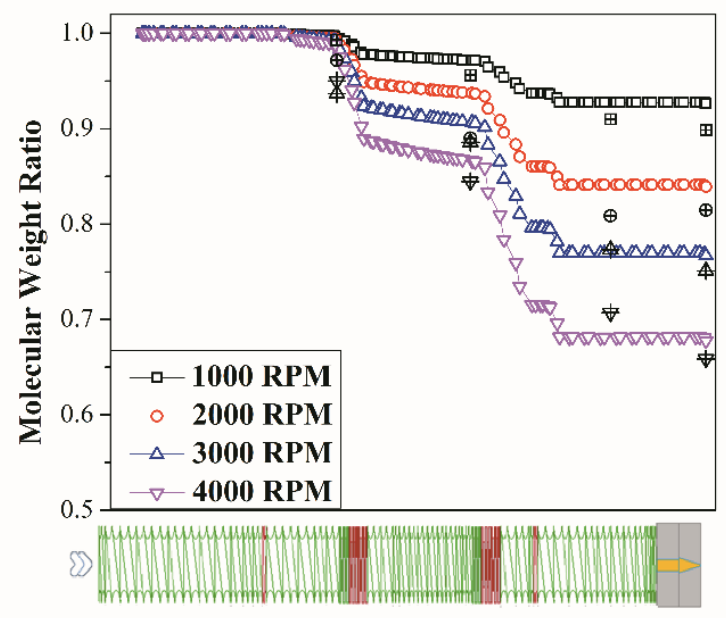

c)

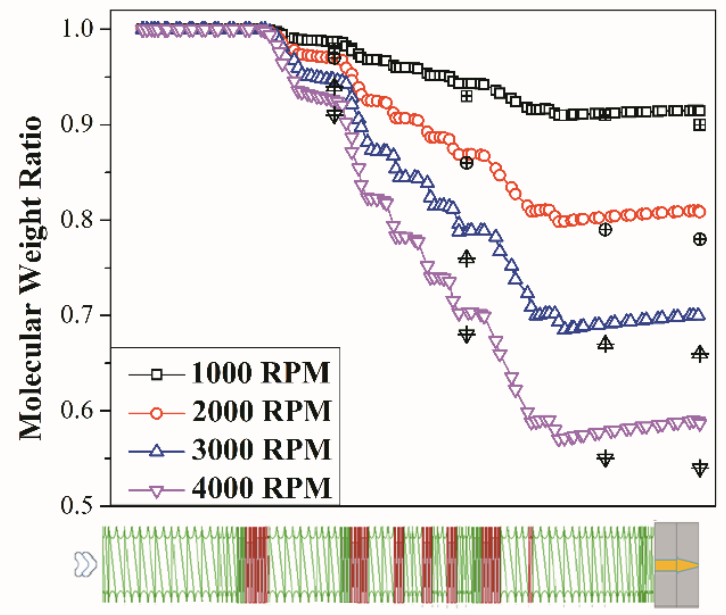

FIGURE 6. Theoretical depropagation reaction ratio (lined plot) and experimental $M_{w} / M_{w 0}$ (single dots) along the barrel axis at various screw speeds for a) smooth Profile 1, b) medium Profile 2, and c) aggressive Profile 3. 
The simulated plots in Figure 6 indicate that the reaction starts upon complete melting of polymer in front of the first restrictive element in the feeding section. The reaction extent is more severe and $M_{w}$ reduction is more rapid at the restrictive elements. This is because both temperature and residence time increase more sharply at the kneading sections. The increasing thermal and mechanical energy input accelerates the reaction and causes more significant drop in $M_{w}$ ratio at the restrictive elements. This effect is more pronounced at higher screw speeds.

The experimental molecular weight measurements are in good agreement with predictions from the theoretical model and simulated flow conditions for all the screw speeds and screw profiles, which validated that our model was able to accurately describe the evolution of conversion along the ultra-high speed extrusion. This validation makes the model useful for practical scale-up problems and optimization of the process, particularly at high speeds where depolymerization is a concern.

\subsection{Mixing Efficiency Model}

During blending and compounding operations, the morphology of the blend system will vary along the screw profile based on the flow and reaction conditions. In this work, we used the calculations of the thermomechanical flow parameters from the simulation to predict mixing efficiency in the ultra-high speed TSE. Two 
important processing parameters that affect the mixing performance are screw speed and residence time. By varying screw speed, one can provide the required amount of mechanical shear rate and shear stress for better dispersion or distribution. In addition, by varying feeder rate one is able to control the residence time inside the extruder in order to achieve a desirable level of mixing, depending on the difficulty of aggregate breakup or other controlling factors. As a result, the evolution of mixing during the processing can be represented numerically by multiplying the local shear rate and the local residence time [32-34]. In our work, we showed this relationship as follow:

Mixing Efficiency :

$\left(\sum \dot{\gamma}_{i} \times x_{i} / \sum x_{i}\right) \times\left(\sum \dot{t}_{i} \times x_{i} / \sum x_{i}\right)$

where $\dot{\gamma}$ in $\mathrm{s}^{-1}$ is the local shear rate within an element of length $x_{i}$ and $t_{i}$ in sec is the residence time at barrel position of $x_{i}$. The mixing efficiency is then a dimensionless parameter, which assesses the extent of mixing along the extruder profile, and should qualitatively track dispersion/distribution or intercalation/exfoliation level in the final blend system. Figure 7 depicts the evolution of mixing along the ultra-high speed TSE for various screw profiles (at a constant screw speed of 2000 RPM and feeder rate of $27 \mathrm{~g} / \mathrm{min})$. As expected, the same screw profile that 
yields greatest polymer degradation is also predicted to improve mixing. Thus, some optimization of the system is needed for practical mixing processes.

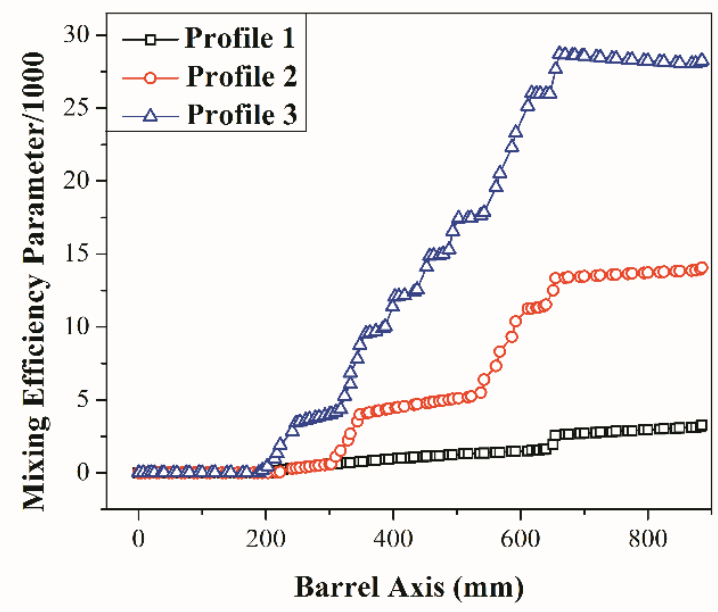

FIGURE 7. Development of mixing efficiency along the ultrahigh speed TSE for various screw profiles.

These theoretical calculations will lead to a predictive characterization of the high-speed extrusion process. Thereby one can decide on the optimal processing conditions and optimal screw program in order to control degradation along the extruder while having high enough shear stress and residence time for an adequate compounding and blending performance, which is the main purpose of using a TSE. Future work will relate the morphological parameters of a blend system to the reactive processing model to predict the local phase morphology changes along the length of the screw during compounding in the high-speed TSE. 


\section{CONCLUSIONS}

In the present work, a kinetic model was derived to predict the evolution of molecular weight ratio reduction through the length of the ultra-high speed twin screw extruder. Two different kinetic constants were introduced in order to take into account the effect of extremely high mechanical shear rate due to processing at high speeds. LUDOVIC ${ }^{\circledR}$ TSE simulation software provided local thermomechanical flow parameters namely temperature, residence time, and shear rate. The model has been validated through comparisons with experiments carried out at different high screw speeds (1000-4000 RPM) and at three different screw profiles (smooth profile, medium profile, and aggressive profile). Samples were collected at three specific locations along the barrel as well as at the die exit for GPC measurements. In all cases, good agreement was found between the theoretical and experimental results. Although we know that the rheological parameters can also change with molecular weight, we assumed that the unprocessed power law relationship held closely enough to estimate the correct viscous dissipation energy. This served as an input to our molecular weight loss model. As evidenced by Figure 6c, actually our model underestimates the extent of degradation. If we were to incorporate a dynamic viscosity that changed with molecular weight as well, our model would predict even less degradation due 
to reduced viscous dissipation with lower viscosity melt. Thus, while some complexities of the physical situation may not be captured, we believe the agreement between experiment and simulation is still remarkable. This work will enable minimizing of the degradation inside the extruder while improving mixing efficiency, which is the main function of ultra-high speed TSE in the plastics industry.

\section{ACKNOWLEDGMENTS}

The authors would like to thank Technovel Corporation of Japan for donation of and assistance with their equipment. Also, we thank Professor Robert Malloy for his help with the high speed twin screw extrusion.

\section{FUNDING}

This work was supported by the National Science Foundation (NSF) (CMMI-1350445). 


\section{REFERENCES}

1. C. Rauwendaal, Polymer extrusion, Carl Hanser Verlag GmbH Co KG, 2014.

2. H.F. Giles Jr, E.M. Mount III, J.R. Wagner Jr, Extrusion: the definitive processing guide and handbook, William Andrew, 2004.

3. M. Xanthos, Reactive extrusion: principles and practice, Hanser Publishers; Distributed in the USA and Canada by Oxford University Press, Munich; New York; New York, 1992.

4. K. Kohlgrüber, W. Wiedmann, Co-rotating twin-screw extruders: Fundamentals, technology, and applications, 2007.

5. W. Michaeli, A. Greefenstein, U. Berghaus, Twin-screw extruders for reactive extrusion, Polym. Eng. Sci. 1995; (35): $1485-1504$.

6. J.L. White, Twin screw extrusion, Technologies and principles, Munich, 1990.

7. A. Farahanchi, E. Boehm, N. Orbey, R. Malloy, The effect of ultra-high speed twin screw extrusion on ABS/organoclay nanocomposite blend properties, Polym. Eng. Sci. 2016; doi:10.1002/pen.24385.

8. J. da R. Silvano, S.A. Rodrigues, J. Marini, R.E.S. Bretas, S.V. Canevarolo, B. de M. Carvalho, L.A. Pinheiro, Effect of 
reprocessing and clay concentration on the degradation of polypropylene/montmorillonite nanocomposites during twin screw extrusion, Polym. Degrad. Stab. 2013; (98): 801-808.

9. C. Remili, M. Kaci, A. Benhamida, S. Bruzaud, Y. Grohens, The effects of reprocessing cycles on the structure and properties of polystyrene/Cloisite15A nanocomposites, Polym. Degrad. Stab. 2011; (96): 1489-1496.

10. L.T. Saw, D.N. Uy Lan, N.A.A. Rahim, A.W. Mohd Kahar, C.X. Viet, Processing degradation of polypropylene-ethylene copolymer-kaolin composites by a twin-screw extruder, Polym. Degrad. Stab. 2015, (111): 32-37.

11. M. Yousfi, S. Lepretre, J. Soulestin, B. Vergnes, M.-F. Lacrampe, P. Krawczak, Processing-induced degradation of nanoclay organic modifier in melt-mixed PET/PE blends during twin screw extrusion at industrial scale: Effect on morphology and mechanical behavior, J. Appl. Polym. Sci. 2014; (131).

12. I.O. Mohamed, R.Y. Ofoli, R.G. Morgan, Modeling the average shear rate in a co-rotating twin screw extruder, $J$. Food Process Eng. 1990; (12): 227-246.

13. K. Eise, H. Herrmann, S. Jakopin, U. Burkhardt, H. Werner, An analysis of twin-screw extruder mechanisms, Adv. Polym. Technol. 1981; (1): 18-39. 
14. R.E. Altomare, P. Ghossi, An analysis of residence time distribution patterns in a twin screw cooking extruder, Biotechnol. Prog. 1986; 2 (1986) 157-163.

15. B. Vergnes, F. Berzin, Modeling of reactive systems in twinscrew extrusion: challenges and applications, Comptes Rendus Chim. 2006; (9): 1409-1418.

16. O.S. Carneiro, J.A. Covas, B. Vergnes, Experimental and theoretical study of twin-screw extrusion of polypropylene, $J$. Appl. Polym. Sci. 2000; (78): 1419-1430.

17. B. Vergnes, G.D. Valle, L. Delamare, A global computer software for polymer flows in corotating twin screw extruders, Polym. Eng. Sci. 1998; (38): 1781-1792.

18. F. Berzin, B. Vergnes, Transesterification of ethylene acetate copolymer in a twin Screw Extruder: experimental and theoretical approaches, Int. Polym. Process. 1998; (13): 1322.

19. A. Poulesquen, B. Vergnes, P. Cassagnau, J. Gimenez, A. Michel, Polymerization of $\in$-caprolactone in a twin screw extruder: experimental study and modelling, Int. Polym. Process. 2001; (16): 31-38.

20. F. Berzin, A. Tara, L. Tighzert, B. Vergnes, Computation of starch cationization performances by twin-screw extrusion, Polym. Eng. Sci. 2007; (47): 112-119. 
21. F. Berzin, B. Vergnes, S.V. Canevarolo, A.V. Machado, J.A. Covas, Evolution of the peroxide-induced degradation of polypropylene along a twin-screw extruder: experimental data and theoretical predictions, J. Appl. Polym. Sci. 2006; (99): 2082-2090.

22. F. Berzin, B. Vergnes, P. Dufoss, L. Delamare, Modeling of peroxide initiated controlled degradation of polypropylene in a twin screw extruder, Polym. Eng. Sci. 2000; (40): 344-356.

23. B. Vergnes, F. Berzin, Peroxide-controlled degradation of poly(propylene): rheological behaviour and process modelling, Macromol. Symp. 2000; (158): 77-90.

24. P.G. Lafleur, B. Vergnes, Polymer Extrusion, John Wiley \& Sons, Ltd, Chichester, UK, 2014.

25. A. Gaspar-Cunha, J.A. Covas, eds., Optimization in polymer processing, Nova Science Publishers, Hauppauge, N.Y, 2011.

26. A. Farahanchi, R. Malloy, M.J. Sobkowicz, Effects of ultrahigh speed twin screw extrusion on the thermal and mechanical degradation of polystyrene, Polym. Eng. Sci. 2016; (56): 743-751.

27. B. Vergnes, F. Berzin, Modelling of flow and chemistry in twin screw extruders, Plast. Rubber Compos. (2004; 33): 409-415. 
28. F. Berzin, B. Vergnes, J. Beaugrand, Evolution of lignocellulosic fibre lengths along the screw profile during twin screw compounding with polycaprolactone, Compos. Part Appl. Sci. Manuf. 2014; (59): 30-36.

29. A. Poulesquen, B. Vergnes, A study of residence time distribution in co-rotating twin-screw extruders. Part I: Theoretical modeling, Polym. Eng. Sci. 2003; (43): 18411848.

30. M. Suparno, K.D. Dolan, P.K.W. Ng, J.F. Steffe, Average shear rate in a twin-screw extrusion as a function of degree of fill, flow behavior index, screw speed and screw configuration: average shera rate in extruder, J. Food Process Eng. 2011; (34): 961-982.

31. J.Y. Cha, M. Suparno, K.D. Dolan, P.K.W. Ng, Modeling thermal and mechanical effects on retention of thiamin in extruded foods, J. Food Sci. 2003; (68): 2488-2489.

32. N. Watzeels, K. Van Durme, H.E. Miltner, C. Block, G. Van Assche, B. Van Mele, B. Bogdanov, H. Rahier, Optimization of extrusion parameters for preparing PCL-layered silicate nanocomposites supported by modeling of twin-screw extrusion, Macromol. Mater. Eng. 2013; (298): 210-220.

33. W. Lertwimolnun, B. Vergnes, Influence of screw profile and extrusion conditions on the microstructure of 
polypropylene/organoclay nanocomposites, Polym. Eng. Sci. 2007; (47): 2100-2109.

34. W. Lertwimolnun, B. Vergnes, Effect of processing conditions on the formation of polypropylene/organoclay nanocomposites in a twin screw extruder, Polym. Eng. Sci. 2006; (46): 314-323. 


\section{FIGURE CAPTIONS}

FIGURE 1. Extrusion set-ups and sampling locations for various screw profiles.

FIGURE 2. Comparison between computed (solid symbols) and measured (open symbols) of a) exit melt temperature and b) minimum residence time at various screw profiles.

FIGURE 3. Temperature profile along different screw profiles at a constant feeder rate and screw speed.

FIGURE 4. RTD for different screw profiles at a constant feeder rate and constant screw speed.

FIGURE 5. Total position average shear rate changes as a function of screw speed at various profiles.

FIGURE 6. Theoretical depropagation reaction ratio (lined plot) and experimental $M_{w} / M_{w 0}$ (single dots) along the barrel axis at various screw speeds for a) smooth Profile 1, b) medium Profile 2, and c) aggressive Profile 3.

FIGURE 7. Development of mixing efficiency along the ultrahigh speed TSE for various screw profiles.

\section{TABLE CAPTIONS}

TABLE 1. Extruder constant values for various screw profiles. 